\title{
Flaxseed Polysaccharide Altering Colonic Gene Expression of Lipid Metabolism and Energy Metabolism in Obese Rat
}

\author{
Xiaohong Lin \\ Jinan University \\ Jiamei Qi \\ Jinan University \\ Guangwen Zhang \\ Jinan University \\ Xichun Peng ( $\nabla$ tpxchun@jnu.edu.cn ) \\ Jinan University https://orcid.org/0000-0002-4804-5203
}

\section{Short report}

Keywords: Flaxseed polysaccharide, RNA-seq, qRT-PCR, colon, obesity

Posted Date: August 18th, 2020

DOI: https://doi.org/10.21203/rs.3.rs-60860/v1

License: (c) (1) This work is licensed under a Creative Commons Attribution 4.0 International License. Read Full License 


\section{Abstract}

\section{Background}

Obesity is one of the most serious public health challenges. Recently, we found that flaxseed polysaccharide (FP) had an anti-obesity effect through promoting lipid metabolism, inducing satiety and regulating gut microbiota, but how FP promote lipid metabolism through altering the colonic epithelial cells remains to be elucidated. In this study, a transcriptome study was performed to investigate the effect of FP altering the gene expression of colonic epithelial cells in an obese rat model.

Results

The transcriptome analysis showed that 3,785 genes were differentially expressed after FP intervention in colonic epithelial cells, including 374 down-regulated and 3,411 up-regulated genes. Through KEGG analysis, we found out three classical pathways related to lipid metabolism and energy metabolism, including PPAR signaling pathway, nitrogen metabolism and oxidative phosphorylation (OXPHOS). Moreover, qRT-PCR results showed consistent expression trends of differential genes with transcriptome analysis.

\section{Conclusions}

The anti-obesity effect of FP may be achieved by regulating the expression of lipid metabolism- and energy metabolism-related proteins acting on the PPAR (peroxisome proliferator-activated receptor) signaling pathway, nitrogen metabolism and OXPHOS pathway in vivo.

\section{Introduction}

Caused by the complex interactions between genes and environmental factors, including food components, diet and/or way of life, obesity is one of the most serious public health challenges facing the world [1]. Over 340 million children and adolescents aged 5-19 in 2016, as well as an estimated 38.2 million children under the age of 5 years in 2019, were overweight or obese [2]. Developing from an imbalance between energy expenditure and energy intake, obesity is a risk factor for the development of several clinical conditions, such as diabetes, cancers and cardiovascular disease [3].

Defined as the edible parts of plants or analogous carbohydrates, dietary fiber resists absorption and digestion in the small intestine, partially or completely fermented in the large intestine [4]. Dietary fiber is beneficial to weight control, mainly through influencing satiation and satiety to control appetite [5]. Besides, fiber intake also has a positive effect on the treatment of pathologies associated with obesity, namely dyslipidemia, hyperglycemia and/or hypertension [6].

Dietary fibers regulated gene expression in colonic epithelial cells and induced genes involved in the processes such as OXPHOS, TCA cycle, glycolysis and electron transport chain, all pathways responsible for generating energy by substrate oxidation [7]. Besides, PPARY is identified as central regulator of 
transcriptional responses to fermented fibers, which regulates genes involved in energy metabolism, in particularly lipid catabolism [7]. What's more, intestinal microbiota can affect both sides of the energy balance equation, as a factor affecting the harvest of energy from dietary ingredients, and as a factor affecting host genes that regulate energy consumption and storage, for example, they can diminish mitochondrial fatty acid oxidation, suppress intestinal epithelial production of Angptl4 to facilitate lipid storage in adipose tissues, show greater inhibition of Cpt1 and so on [8].

In the previous research, we had found flaxseed polysaccharides (FP) had an anti-obesity effect via promoting lipid metabolism, inducing satiety and regulating the intestinal flora [9-10]. However, as a polysaccharide, FP can not directly enter the body to play a role, so we speculate that it can promote weight loss through the interaction with intestinal flora, change the gene expression of intestinal epithelial tissue, and induce the metabolism of fat and energy in vivo. However, it is not clear which intestinal epithelial tissue gene expression is changed by flaxseed polysaccharide. In the present study, we aimed to find out the significantly different genes affected by FP involved in lipid and energy metabolism in colonic epithelial cells, further investigating the mechanism of FP in weight loss.

\section{Materials And Methods}

\section{Animals, diets, and sample preparation}

18 male Sprague-Dawley (SPF grade), 4 weeks old, were bought from Guangdong Medical Laboratory Animal Center (Guangzhou, China.). Group Con $(n=6)$ was fed with a standard diet (D12450B), others were given high-fat diet (D12492) to establish the obesity model, which were finally divided into two groups, Group HFD and Group FPD (6 rats per group). After that, Group Con and Group HFD were fed with the control diet, AIN-93M (3.77 kcal/g), while Group FPD was fed with a 10\% FP diet, replaced 10\% corn starch (in AIN-93M) with equivalent FP $(3.37 \mathrm{kcal} / \mathrm{g})$. The rats were weighed weekly. The details of the diets in this research are as labeled in Table 1. 
Table 1

Detailed Recipes of Each Diet (\%)

\begin{tabular}{|lllll|}
\hline Ingredients & $\begin{array}{l}\text { standard } \\
\text { diet } \\
\text { (D12450B) }\end{array}$ & $\begin{array}{l}\text { high-fat } \\
\text { diet }\end{array}$ & $\begin{array}{l}\text { control } \\
\text { diet (AIN- }\end{array}$ & $\begin{array}{l}\text { flaxseed } \\
\text { polysaccharide }\end{array}$ \\
\hline $\begin{array}{l}\text { 93M) } \\
\text { flaxseed }\end{array}$ & 0.00 & 0.00 & 0.00 & 10.00 \\
polysaccharide & & & & \\
\hline corn starch & 33.00 & 0.00 & 46.57 & 36.57 \\
\hline dextrin & 3.35 & 16.35 & 15.50 & 15.50 \\
\hline casein & 19.13 & 26.17 & 14.00 & 14.00 \\
\hline sucrose & 34.47 & 9.00 & 10.00 & 10.00 \\
\hline cellulose & 4.78 & 6.54 & 5.00 & 5.00 \\
\hline soybean oil & 2.39 & 3.27 & 4.00 & 4.00 \\
\hline lard & 1.91 & 32.06 & 0.00 & 0.00 \\
\hline mineral mix ain-93 & 3.35 & 4.58 & 3.50 & 3.50 \\
\hline vitamin mix ain-93 & 0.96 & 1.31 & 1.00 & 1.00 \\
\hline L-cystine & 0.29 & 0.39 & 0.18 & 0.18 \\
\hline choline bitartrate & 0.24 & 0.33 & 0.25 & 0.25 \\
\hline
\end{tabular}

On day 53, all rats were fasted overnight and were anesthetized with sodium pentobarbital. After execution of the rats, the colonic epithelial cells of each rat were scraped. They were frozen in liquid nitrogen shortly upon sampling and then stored at $-80^{\circ} \mathrm{C}$ until RNA extraction. The experiments were ratified by the Research Animal Administration Center of Jinan University (Guangzhou, China), following all Institutional Animal Care and Use Committee of Jinan University guidelines for the use and care of animals.

\section{RNA extraction}

TRIzol reagent (Invitrogen, CA, USA) was used to extract the total RNA of rats ( $\mathrm{n}=6$ per group) from the colonic epithelial cells according to the manufacturer's instructions. Genomic DNA was removed by Deoxyribonuclease I (TaKara, Dalian, China). We determined the RNA quality with a 2100 Bioanalyzer (Agilent, Santa Clara, CA, USA) and quantified it by ND-2000 (Thermo Fisher, Shanghai, China). The sequencing library was constructed by using high-quality RNA samples $(O D 260 / 230 \geq 2.0,0 D 260 / 280=$ $1.8 \sim 2.2$, RNA integrity number $\geq 6.5,>10 \mu \mathrm{g}, 28 \mathrm{~S}: 18 \mathrm{~S} \geq 1.0$ ) were used to construct the sequencing library. 


\section{Library preparation and Illumina HiSeq 4,000 sequencing}

First, messenger RNA was isolated by using oligo (dT) beads according to the polyA selection method, fragmented by fragmentation buffer. Then, double-stranded cDNA was synthesized by using Super Script double-stranded cDNA synthesis Kit (Invitrogen, CA, USA) and random hexamer primers (Illumina, Shanghai, China). A library of 200-300 bp cDNA target fragments was selected on $2 \%$ Low Range Ultra Agarose (Bio-Rad, CA, USA), and then PCR amplification of 15 PCR cycles was carried out using Phusion DNA polymerase (NEB, Beijing, China). After the quantification with TBS380, we sequenced the paired-end RNA-Seq sequencing library with Illumina HiSeq 4000 (sequencing mode of paired-end, $2 \times 126 \mathrm{bp}$ ).

\section{Read mapping}

The raw paired-end reads were trimmed and the default parameters were used for quality control with SeqPrep (https://github.com/jstjohn/SeqPrep) and Sickle (http://www.ensembl.org/Rattus_norvegicus/Info/Index). Then, bowtie2 (http://bowtiebio.sourceforge.net/index.shtml) software was used to accurately compare clean reads with reference genome (http://www.ensembl.org/Rattus_norvegicus/Info/Index) through orientation mode. The mapping standard of bowtie was that the sequencing reading should uniquely match the genome, allowing up to two mismatches, and no insertions or deletions. Besides, the whole genome was divided into $15 \mathrm{kbp}$ windows, sharing $5 \mathrm{kbp}$. The new transcription regions were defined as more than two consecutive windows without an overlapping gene region. There were at least two reads mapped per window in the same orientation. The sequencing data were annotated with the databases Ensemble, KEGG, GO, eggNOG, UniProtKB and Symbol.

\section{Differential expression analysis and functional enrichment}

In order to identify the DEGs among several different samples, we calculating the expression level of each transcript by the fragments per kilobase of exon per million mapped reads (FRKM) method. RSEM (RNASeq by Expectation-Maximization, http://deweylab.biostat.wisc.edu/rsem/) was used to quantify gene abundance and utilized DESeq2 (http://bioconductor.org/ packages/stats/bioc/DESeq2/) was used for differential expression analysis. When the adjusted P-value (Benjamin Hochberg (BH) multiple test correction method) was less than 0.05 , it was considered that genes were significant differentially expressed. The difference of gene expression was observed by volcano map. KEGG (Kyoto Encyclopedia of Genes and genome database) pathway analyses and KEGG functional enrichment were performed. It was considered to be a significantly different expression level if the false discovery rate (FDR) was less than 0.05 in the multi-group comparison. By using the BLAST algorithm (blastx/blastp2.2.24+), all the genes were mapped to the KEGG gene database (GENES) to find the potential biological pathway of the genes.

\section{Quantitative real-time PCR (qRT-PCR) analysis}

TRIzol reagents (Invitrogen, CA, USA) were used to extract total RNA. Following the supplier's instructions, the cDNA was synthesized with a BestarTM qPCR RT kit (DBI Bioscience, Shanghai, China). Then, the 
gene mRNA expression was determined with a BestarTM SybrGreen qPCR Master Mix. Briefly, a $20 \mu \mathrm{L}$ qPCR mixture was prepared as follows: $0.5 \mu \mathrm{L}$ of reverse primer $(10 \mu \mathrm{mol} / \mathrm{L}), 0.5 \mu \mathrm{L}$ of forward primer $(10 \mu \mathrm{mol} / \mathrm{L}), 1 \mu \mathrm{L}$ of cDNA, $8 \mu \mathrm{L}$ of double-distilled water and $10 \mu \mathrm{L}$ of Bestar ${ }^{\circledR}$ SybrGreen qPCR Master Mix (DBI Bioscience, Shanghai, China). Real-time PCR was performed with a Stratagene Mx3000p Real time PCR system (Agilent, Santa Clara, CA, USA). The PCR conditions were initially maintained at $95^{\circ} \mathrm{C}$ for $2 \mathrm{~min}$, followed by 40 cycles of $94^{\circ} \mathrm{C}$ for $20 \mathrm{~s}, 58^{\circ} \mathrm{C}$ for $20 \mathrm{~s}$ and $72{ }^{\circ} \mathrm{C}$ for $20 \mathrm{~s}$. Primers were designed using Primer 5 and the sequences of all the primers used are provided in Supplementary Table S1. The average cycle threshold (Ct) values were used for quantification using the $2^{-\Delta \Delta \mathrm{Ct}}$ method, with $\beta$ actin as reference gene.

\section{Statistical Analysis}

Numerical data were presented as means \pm standard deviation (SD). The difference between means was analyzed using SPSS 25.0 software (IBM Corporation, Armonk, New York). Two-tailed Student's t-tests were conducted to compare the data in different groups. Besides, $\mathrm{P}<0.05\left({ }^{*}\right)$ or $\left.\mathrm{P}<0.01{ }^{(\star *}\right)$ was considered as statistically significant.

\section{Results}

\section{Gene expression profile of rat colonic epithelial cells}

As showed in our previous research, the body weight of rats in Group FPD was significantly lower than that of group HFD, and finally became very similar to those in Group Con at the end of the trial, which indicated that FP induced body weight loss [9]. Approximately 52.16 million raw reads were obtained from three groups. A huge proportion (95.14\%) of high-quality clean reads was generated after adapter trimming and filtering low-quality reads. The percentages of clean reads having a base quality greater or equal than Q30 were above $95.14 \%$ with $48.37-50.25 \%$ of GC content, indicating that the data produced by sequencing are of high quality. On average, $96.04 \%$ of clean reads were mapped to the reference genome, among which $90.06 \%$ were aligned to unique locations.

As shown in Fig. $1 \mathrm{a}, 80.45 \%$ of the genes were in common among all three groups. Besides, the gene composition between Group Con and Group HFD were somewhat similar, while Group FPD was obviously different compared with them (Fig. 1b). This result suggested that FP intervention would change the gene composition of colonic epithelial tissues.

\section{Identification of Differentially Expressed Genes}

The volcano plot showed the variation of mRNA expression between Group HFD and Group Con (Fig. 2a). In total, 28 DEGs, including 14 upregulated and 14 downregulated, were identified. Besides, 3,785 DEGs were identified between Group HFD and Group FPD (Fig. 2b), including 374 downregulated and 3411 upregulated DEGs $(P<0.05)$. Compared with Group HFD, there were a lot of DEGs in Group FPD, but few in Group Con. Therefore, our subsequent analysis would focus on Group HFD and Group FPD. 


\section{KEGG Pathway Analysis of Differentially Expressed Genes}

The DEGs were mapped in the KEGG pathway database. Compared with Group HFD and Group FPD, the annotated genes were classified into six categories. Most of them were enriched in signal transduction. Besides, 15 DEGs were enriched in the pathway of Energy metabolism and 26 DEGs in PPAR signaling pathway (Fig. 3).

\section{KEGG Pathway Enrichment Analysis of Differentially Expressed Genes}

It has been reported that weight loss involves a variety of mechanisms, such as inhibiting energy intake, stimulating energy expenditure and so on. Thus, KEGG pathway enrichment analysis of 15 DEGs in 'Energy metabolism' between Group HFD and Group FPD was performed. As a result, 13 metabolic pathways with significantly differential expressions $(p<0.05)$ were identified between Group HFD and Group FPD, of which 10 pathways showed the most significant difference $(p<0.01)$. Figure 4 shows the top 13 ranked KEGG pathway of DEGs. Nitrogen metabolism occupied the strongest enrichment degree as it possessed the highest Rich factor, followed by monobactam biosynthesis. Nitrogen metabolism had the most DEGs, and the then is OXPHOS. The DEGs in the two pathways were listed in Table 2, and all of them were upregulated. Especially, there were 9 genes of carbonic anhydrase enriched in nitrogen metabolism. 
Table 2

Information of three KEGG pathways and DEGs enriched

\begin{tabular}{|c|c|c|c|}
\hline KEGG pathway & $\begin{array}{l}\text { Gene } \\
\text { name }\end{array}$ & Gene description & Regulation \\
\hline \multirow[t]{4}{*}{$\begin{array}{l}\text { Oxidative } \\
\text { phosphorylation }\end{array}$} & Ndufa4I2 & $\begin{array}{l}\text { NDUFA4, mitochondrial complex associated } \\
\text { like } 2\end{array}$ & Upregulated \\
\hline & Atp6v1g2 & ATPase $\mathrm{H}+$ transporting V1 subunit G2 & Upregulated \\
\hline & Cox4i2 & cytochrome c oxidase subunit $4 \mathrm{i} 2$ & Upregulated \\
\hline & Cox6b2 & $\begin{array}{l}\text { cytochrome c oxidase subunit VI b } \\
\text { polypeptide } 2\end{array}$ & Upregulated \\
\hline \multirow[t]{10}{*}{ Nitrogen metabolism } & Cps1 & carbamoyl-phosphate synthase 1 & Upregulated \\
\hline & Car3 & carbonic anhydrase 3 & Upregulated \\
\hline & Car1 & carbonic anhydrase I & Upregulated \\
\hline & $\mathrm{Ca} 5 \mathrm{~b}$ & carbonic anhydrase $5 \mathrm{~B}$ & Upregulated \\
\hline & Car8 & carbonic anhydrase 8 & Upregulated \\
\hline & Car13 & carbonic anhydrase 13 & Upregulated \\
\hline & Car2 & carbonic anhydrase 2 & Upregulated \\
\hline & Car12 & carbonic anhydrase 12 & Upregulated \\
\hline & Car14 & carbonic anhydrase 14 & Upregulated \\
\hline & Car4 & carbonic anhydrase 4 & Upregulated \\
\hline \multirow{10}{*}{$\begin{array}{l}\text { PPAR signaling } \\
\text { pathway }\end{array}$} & Plin4 & perilipin 4 & Upregulated \\
\hline & Fabp1 & fatty acid binding protein 1 & Upregulated \\
\hline & Fabp3 & fatty acid binding protein 3 & Upregulated \\
\hline & Cyp8b1 & $\begin{array}{l}\text { cytochrome P450, family } 8 \text {, subfamily b, } \\
\text { polypeptide } 1\end{array}$ & Upregulated \\
\hline & Scd & stearoyl-CoA desaturase & Upregulated \\
\hline & Fabp4 & fatty acid binding protein 4 & Upregulated \\
\hline & Lpl & lipoprotein lipase & Upregulated \\
\hline & Acsl6 & $\begin{array}{l}\text { acyl-CoA synthetase long-chain family } \\
\text { member } 6\end{array}$ & Upregulated \\
\hline & Plin1 & perilipin 1 & Upregulated \\
\hline & Pltp & phospholipid transfer protein & Upregulated \\
\hline
\end{tabular}




\begin{tabular}{|c|c|c|c|}
\hline KEGG pathway & $\begin{array}{l}\text { Gene } \\
\text { name }\end{array}$ & Gene description & Regulation \\
\hline & Angpt|4 & angiopoietin-like 4 & Upregulated \\
\hline & Rxrg & retinoid $\mathrm{X}$ receptor gamma & Upregulated \\
\hline & Adipoq & $\begin{array}{l}\text { adiponectin, C1Q and collagen domain } \\
\text { containing }\end{array}$ & Upregulated \\
\hline & Acsbg2 & $\begin{array}{l}\text { acyl-CoA synthetase bubblegum family } \\
\text { member } 2\end{array}$ & Upregulated \\
\hline & Fabp5 & fatty acid binding protein 5 , epidermal & Upregulated \\
\hline & Fabp2 & fatty acid binding protein 2 & Upregulated \\
\hline & Aqp7 & aquaporin 7 & Upregulated \\
\hline & Cpt1c & carnitine palmitoyl transferase $1 \mathrm{c}$ & Upregulated \\
\hline & Acs 14 & $\begin{array}{l}\text { acyl-CoA synthetase long-chain family } \\
\text { member } 4\end{array}$ & Upregulated \\
\hline & Pparg & $\begin{array}{l}\text { peroxisome proliferator-activated receptor } \\
\text { gamma }\end{array}$ & Upregulated \\
\hline & Acsl1 & $\begin{array}{l}\text { acyl-CoA synthetase long-chain family } \\
\text { member } 1\end{array}$ & Upregulated \\
\hline & Acsbg1 & $\begin{array}{l}\text { acyl-CoA synthetase bubblegum family } \\
\text { member } 1\end{array}$ & Downregulated \\
\hline & Gk & glycerol kinase & Downregulated \\
\hline
\end{tabular}

\section{Expression correlation analysis and Protein-Protein Interaction (PPI) Network Analysis of DEGs}

The DEGs enriched in PPAR signaling pathway were further analyzed. Based on the correlation of gene expression, the correlation coefficient between gene and gene is obtained by Spearman algorithm. To systemically analyze the functions of these DEGs, we mapped them to PPI data and obtained an PPI network (Fig. 5). The network showed the association between 23 DEGs (Table 2). The result suggested that the upregulation of Pparg, Lpl, Fabp1 and Acsl1 might play an important role in colonic epithelial tissues.

\section{Verification of differentially expressed genes}

In order to verify the results of transcriptome sequencing, 17 important DEGs were chosen for quantitative RT-PCR. The results of qRT-PCR are shown in Fig. 6. The expression trends were consistent with those obtained by RNA-seq, suggesting that the RNA-seq datas reliably reflected the gene expression alterations. 


\section{Discussion}

This experiment investigated the gene expression of colonic epithelial cells affected by FP intervention in an obese rat model. After FP intervention, 3,785 DEGs were found in the obese rats, 15 of which were enriched in energy metabolism. Among them, 10 DEGs were enriched in nitrogen metabolism, including 9 genes of carbonic anhydrase and Cps1. Four DEGs were enriched in OXPHOS, including Cox4i2, Cox6b2, Atp6v1g2 and Ndufa4I2. Besides, 23 DEGs in PPAR signaling pathway showed strong connection.

As showed in our previous research, obese rats in Group FPD showed the reduction of body weight loss as well as body fat [9]. It is well-known that obesity is always related to an imbalance between the accumulation and consumption of lipids and energy. Thus, since FP intervention had been proved to lose weight, we speculated that it could improve lipid metabolism and energy metabolism. Our present results were consistent with our previous report, and more importantly, we found that FP intervention did improve the lipid metabolism, mainly by regulation of the PPAR signaling pathway in colonic epithielial tissues.

\section{FP intervention promoted lipid metabolism of the colonic epithelial tissue}

Most of the dietary lipids are long-chain TGs, which are hydrolyzed in the intestine to produce FAs and monoglycerides [11]. Then, they will enter enterocytes. As the members of the lipid-binding protein superfamily, FABPs bind to saturated and unsaturated LCFA and other hydrophobic ligands such as monoacylglycerols, mediating FA transportation to different cell organelles [12]. Thus, FP intervention upregulated the expression of Fabp1- Fabp5, heightening the transshipment of FA. After entering enterocytes, FFAs and glycerol arrive at the crossroads of several pathways; they can be metabolized within mitochondria, or be re-esterified to TGs, packaged into chylomicrons (CMs), and secreted from enterocytes to the lymph [13], which results in elevated TG levels in the circulation. CMs are the main contributors to the serum lipid level, and the decreased clearance or increased production of them can lead to hypertriglyceridemia [14]. High intake of dietary fat increases postprandial plasma TG levels significantly, and the regulation of lipid metabolism in intestinal epithelial cells could affect postprandial hyperlipidemia [15]. Most dietary fatty acids are processed as CM-TG, which can be later metabolized at the tissue level by LPL, releasing FA for tissue uptake [16]. Thus, FP intervention upregulated Lpl, inhibiting the release of $\mathrm{CMs}$ and the rise of plasma TG levels.

As a key enzyme in glycerol metabolism, GK catalyzes the conversion of glycerol to glycerol 3-phosphate, which is the main substrate in TG synthesis [17]. As a pore-forming transmembrane protein, the aquaglyceroporin AQP7 facilitates the transport of glycerol across cell membranes [18]. AQP7 involves in rapid fluid movement through the villus epithelium in the small and large intestine [19]. Therefore, FP intervention downregulated Gk to inhibit the synthesis of TG, and upregulated Aqp7 to facilitate the transport of glycerol that would be metabolized in other tissues. 
It is also very important to accelerate the oxidation of FAs, which in turn helps inhibit their reesterification. In the present study, the upregulation of Pparg and Rxrg was found. Several prior studies have demonstrated that administering mice PPAR agonists can induce the expression of lipid oxidation genes and $\beta$ - oxidation in intestinal cells, and inhibit enterocytic lipid accumulation and post-prandial lipidemia [20]. RXR is a heterodimeric partner of PPARs, and LCFA may increase transcriptional activity of PPARs by acting as a physiological ligand of RXR [21]. Researchers found that the activation of PPARY could control pathways connected with FA metabolism and mitochondrial function [13]. Thus, FP intervention enhanced the activation of PPARY and RXR, altering DEGs of colonic epithelial tissue to perform biological responses and transcription of genes related to lipids.

Most cellular FA metabolic pathways need to be preferentially activated by thioesterification, and acylCoA synthetase catalyzes the formation of active FA [22]. ACSLs catalyze the conversion of free longchain fatty acids into their acyl-coenzyme A ( $\mathrm{COA}$ ) forms [23]. The transport of long-chain acyl-CoA esters into the mitochondria matrix is mediated by the CPT system, among which CPT1 is responsible for converting acyl-CoAs into acylcarnitine to shuttle across the mitochondria membranes [24]. Thus, FP intervention upregulated Acsl1, AcsI4, Acsl6 and Cpt1c, which promoted the conversion of FA to acyl-CoA and the start of mitochondrial fatty acid $\beta$-oxidation. Besides, SCFAs and MCFAs permeate the inner mitochondrial membrane in the non-esterified form, which are activated into their CoA-derivatives in the mitochondrial matrix [25]. Afterwards, these FAs participate in $\beta$-oxidation and the TCA cycle. The final products include $\mathrm{CO}_{2}, \mathrm{NADH}$ and $\mathrm{FADH}_{2}$.

In addition to accelerate FA oxidation, FP intervention also heightened cholesterol catabolism. As the key enzyme in the bile acid formation pathway, Cyp8b1 was found to be inhibited expressed in high fat diet mice, which inhibited the catabolism of cholesterol into bile acids, causing the elevated cholesterol [26]. Researchers found the elevation of PLTP activity results in rapid disposal of cholesterol from body through increased conversion into bile acids and subsequent excretion [27]. Thus, FP intervention upregulated Cyp8b1 and Pltp to accelerate cholesterol catabolism. Besides, FP intervention also played an important role in the prevention of inflammation. Two or more members of the perilipin family of lipid droplet surface proteins decorate lipid droplets in chordates [28]. As metabolically dynamic cellular organelles, lipid droplets are specialized in storing free fatty acids, the excess of which have toxic effects and can lead to inflammation [29]. Researchers found that Plin1 deficiency secreted pro-inflammatory lipid metabolites, exacerbating adipose tissue inflammation [29]. The colonic epithelial cells responded to elevated FAs by increasing expression of Perilipin under FP intervention, inhibiting inflammation. Adiponectin is encoded by the Adipoq gene, controls the metabolism of glucose and FA and has a general anti-inflammatory effect [30]. Adiponectin protects against murine colitis and maintains intestinal homeostasis through by modulating adaptive immunity and interactions with its receptor AdipoR1 [31]. Angptl4 protected against acute colonic inflammation and that its absence worsened the severity of inflammation [32]. As the critical enzyme in FA metabolism, SCD is responsible for the conversion of saturated FAs to unsaturated FAs, and oleic acid is one of the major products [33]. Researchers found that gut-specific SCD-1 activity is necessary to control intestinal epithelial inflammation and synthetize 
oleate, and that dietary oleic acid provides protection against intestinal inflammation in vivo [34]. Thus, FP intervention upregulated Plin1, Plin4, Adipoq, Angpt|4 and Scd to regulate and attenuate inflammation.

In conclusion, FP intervention modulated PPAR signaling pathways to suppress triglyceride accumulation mainly by stimulating fatty acid oxidation, as well as reduce inflammation.

\section{FP intervention promoted energy metabolism of the colonic epithelial tissue}

We found plenty of CAs were upregulated. As isozymes, CAs catalyze the carboxylation of water to form carbonic acid, which spontaneously decomposes into protons and bicarbonate $\left(\mathrm{H}_{2} \mathrm{O}+\mathrm{CO}_{2} \leftrightarrow \mathrm{H}_{2} \mathrm{CO}_{3} \leftrightarrow\right.$ $\mathrm{HCO}_{3}{ }^{-}+\mathrm{H}^{+}$) [35]. The CAs we found includes several isoforms: cytosolic (CA I, CA II, CA III, CA XII), membrane-bound (CA IV, CA XII and CA XIV), mitochondrial (CA V) and catalytically inactive (CA VIII) [3638]. The luminal mucus from the colon and stomach of guinea pigs, mice and humans showed a large amount of CA activity, by which the hydration rate of $\mathrm{CO}_{2}$ was increased by 1000-2000 times, about 1 / 10 of the rate found in red cells [39]. Thus, as showed in Fig. 7, FP intervention up-regulated CAs and accelerated the consumption of $\mathrm{CO}_{2}$, reducing the accumulation of it in cells and enhancing the TCA cycle. This would further increased energy consumption.

We also found the upregulation of Ca5b and Cps1. Localized in mitochondria, Cps1 catalyzes the condensation of metabolic ammonia and $\mathrm{HCO}_{3}{ }^{-}$to carbamyl phosphate [40]. Researchers suggested $\mathrm{CA}$ $V$ in enterocytes provided bicarbonate ions to CPS1 and facilitated the conversion of ammonia to citrulline, the first two steps of the urea cycle, which played an important role in detoxification and disposal of ammonia produced in the gut [41]. Approximately $12-18 \mathrm{~g}$ of protein reaches the human colon daily, and the main pathway of amino acid fermentation is deamination, leading to the production of ammonia, most of which is absorbed and metabolized into urea by the liver and excreted in urine [42]. Thus, FP intervention reduced the accumulation of bicarbonate ions in mitochondria, enhancing the initiation of the urea cycle and ammonium removal.

Generated from the TCA cycle and $\beta$-oxidation, NADH and $\mathrm{FADH}_{2}$ provide electrons to the ETC [43]. The electrons are transferred to $\mathrm{O}_{2}$ at complex IV to produce $\mathrm{H}_{2} \mathrm{O}$, and the pumped protons produce an electrochemical gradient on the inner membrane of mitochondria, which is the driving force for complex $\mathrm{V}$ to produce ATP [44]. As a component of the ETC complex I subunit, NDUFA4L2 fine-tunes complex I activity, and mediates mitochondrial activation of OXPHOS [45]. COX6B2 facilitates the assembly of complex IV to support mitochondrial respiration and OXPHOS-induced generation of ATP [46]. We also found the upregulation of ATP6V1G2, which encoded subunit G2 of vacuolar ATPase (V-ATPase), transporting protons from the cytoplasm into the lysosome and maintaining lysosomal acidification [47]. V-ATPases are highly conserved proton pumps, and V1 is the peripheral membrane subcomplex of them, which contains the sites of ATP hydrolysis [48]. Thus, FP intervention upregulated Ndufa4l2 and Cox6b2 to heighten OXPHOS, accelerating the generation of ATP and stimulating energy consumption. Besides, FP intervention also upregulated Atp6v1g2, hydrolyzing ATP to provide energy for $\mathrm{H}^{+}$transport. 
As the TCA progress and oxygen consumption in the ETC increase, however, the oxygen concentration in the colonic epithelial cells decreases. As the major consumers of oxygen in the cells, mitochondria are severely affected by decreased oxygen availability, and thus OXPHOS is adapted to hypoxia by remodeling ETC as well as the activity of the TCA cycle [49]. The COX4 subunit optimizes respiratory chain function according to oxygen-controlled expression of its isoforms COX4i1 and COX4i2, and COX4i2 facilitates a decrease in cytochrome c oxidase affinity to oxygen [50]. Both NDUFA4L2 and COX4I2 can reduce the capacity for mitochondrial oxygen consumption and act to limit mitochondrial ROS production during hypoxia, by reducing the activity of complex I and cytochrome c oxidase respectively [51]. Researchers found the deterioration of respiratory capacity due to differences at the level of respiratory chain complexes in obese individuals, among which, particularly, electron transport at the level of complexes I and IV was most affected [52]. Therefore, FP intervention upregulated Ndufa4I2 and Cox4i2, preventing mitochondria from hypoxia, which kept the energy expenditure steady and healthy.

As we have mentioned above, the Adipoq gene of colonic epithelial tissues that encode adiponectin was upregulated. In the previous researches, we had found FP intervention significantly upregulated adiponectin probably via the gut-brain axis. There is growing evidence that the metabolic benefits of bariatric surgery are the result of complex gastrointestinal regulation, which produces signals that "educate" the brain to adapt to the new gut environment, and these signals somehow lower the defense levels of body fat [53]. Besides, the gut-brain axis also plays a key role in the control of energy balance [54]. Therefore, FP intervention might improve lipid metabolism and energy metabolism via the gut-brain axis to achieve some of the impressive effects of bariatric surgery with medications.

\section{Conclusion}

In summary, this study further sheds light on the mechanisms involved in the reported effects of FP intake. FP intervention had directly or indirectly altered the gene expression in epithelial cells, which further changed the host's lipid metabolism and energy metabolism, finally resulting in weight loss. By altering gene expression of the PPAR signaling pathway in the colonic epithelial cells, FP intervention accelerated FA catabolism to reduce accumulation. By promoting energy metabolism, FP intervention facilitated the transformation of FA oxidation products to ATP. As shown here, this study offers convincing evidence that FP may lose weight by regulating some potential key genes, which are involved in lipid metabolism and energy metabolism. Proving the role of FP in the prevention of obesity and obesity-related chronic diseases, these novel findings may provide a powerful and novel mean to clarify FP as an efficient functional food candidate in therapy of obesity.

\section{Abbreviations}

FP, flaxseed polysaccharide; PPAR, peroxisome proliferator-activated receptor; OXPHOS, oxidative phosphorylation; LPL, Lipoprotein lipase; TG, triglycerides; FA, fatty acid; FABP, fatty acid-binding proteins; DEGs, differentially expressed genes; VLDL, very low density lipoprotein; ACSL, acyl-CoA synthetase 
enzymes specific for long chain; SCD, Stearoyl-CoA desaturase; AQP7, aquaglyceroporin 7; Gyk, Glycerol kinase; Angpt|4, Plasma angiopoietin-like 4; CAs, Carbonic anhydrases; CPS1, Carbamyl phosphate synthetase I; COX6B2, Cytochrome c oxidase subunit 6B2; PLTP, Phospholipid transfer protein; UC, ulcerative colitis; FFA

free fatty acids; LCFA, long chain fatty acids; MCFAs:medium-chain fatty acids; TCA cycle:tricarboxylic acid cycle; ETC:electron transport chain; ROS:reactive oxygen species; CMs:chylomicrons

\section{Declarations}

\section{Acknowledgements}

The authors thank Bing Yu, Ruixia Qiu and Shulin Deng from the Department of Food Science and Engineering, Jinan University, for their valuable contribution to the experiments in this study.

\section{Authors' contributions}

$\mathrm{XL}, \mathrm{JQ}$ and $\mathrm{GZ}$ designed the study, performed research, analyzed data and prepared the manuscript. XP contributed to the scientific idea, supervised the study, and revised the manuscript.

\section{Funding}

This work was supported by the National Natural Science Funds (No. 31801543, No. 81601299, and No. 81800833 ) and the funds of the China Postdoctoral Science Foundation (No. $2018 \mathrm{M} 643368$ and No. 2018M631045).

\section{Availability of data and materials}

All data generated or analyzed during this study are available from the corresponding author upon reasonable request.

\section{Competing interests}

The authors declare that they have no competing interests.

\section{References}

1. Hill JO. Understanding and addressing the epidemic of obesity: an energy balance perspective. Endocr Rev. 2006;27(7):750-61.

2. World Health Organization. Fact sheet: Obesity and overweight. Available from.

3. http://, Accessed Apr 2020.

4. Han SF, Jiao J, Zhang W, Xu JY, Zhang W, Fu CL, et al. Lipolysis and thermogenesis in adipose tissues as new potential mechanisms for metabolic benefits of dietary fiber. Nutrition. 2017;33:11824. 
5. Papathanasopoulos A, Camilleri M. Dietary fiber supplements: effects in obesity and metabolic syndrome and relationship to gastrointestinal functions. Gastroenterology. 2010;138(1):65-72.e722.

6. Slavin J. Fiber and prebiotics: mechanisms and health benefits. Nutrients. 2013;5(4):1417-35. Published 2013 Apr 22.

7. Aleixandre A, Miguel M. Dietary fiber in the prevention and treatment of metabolic syndrome: a review. Crit Rev Food Sci Nutr. 2008;48(10):905-12.

8. Lange K, Hugenholtz F, Jonathan MC, Schols HA, Kleerebezem M, Smidt H, et al. Comparison of the effects of five dietary fibers on mucosal transcriptional profiles, and luminal microbiota composition and SCFA concentrations in murine colon. Mol Nutr Food Res. 2015;59(8):1590-602.

9. Bäckhed F, Manchester JK, Semenkovich CF, Gordon JI. Mechanisms underlying the resistance to diet-induced obesity in germ-free mice. Proc Natl Acad Sci U S A. 2007;104(3):979-84.

10. Luo J, Qi J, Wang W, Luo Z, Liu L, Zhang G, et al. Antiobesity Effect of Flaxseed Polysaccharide via Inducing Satiety due to Leptin Resistance Removal and Promoting Lipid Metabolism through the AMP-Activated Protein Kinase (AMPK) Signaling Pathway. J Agric Food Chem. 2019;67(25):7040-9.

11. Luo J, Li Y, Mai Y, Gao L, Ou S, Wang Y, et al. Flaxseed gum reduces body weight by regulating gut microbiota. J Funct Food. 2018;47:136-42.

12. Van Dyck F, Braem CV, Chen Z, Declercq J, Deckers R, Kim BM, et al. Loss of the PlagL2 transcription factor affects lacteal uptake of chylomicrons. Cell Metab. 2007;6(5):406-13.

13. Amiri M, Yousefnia S, Seyed Forootan F, Peymani M, Ghaedi K, Nasr Esfahani MH. Diverse roles of fatty acid binding proteins (FABPs) in development and pathogenesis of cancers. Gene. 2018;676:171-83.

14. Duszka K, Oresic M, Le May C, König J, Wahli W. PPARy Modulates Long Chain Fatty Acid Processing in the Intestinal Epithelium. Int J Mol Sci. 2017;18(12):2559.

15. Demignot S, Beilstein F, Morel E. Triglyceride-rich lipoproteins and cytosolic lipid droplets in enterocytes: key players in intestinal physiology and metabolic disorders. Biochimie. 2014;96:48-55.

16. Kimura R, Takahashi N, Lin S, Goto T, Murota K, Nakata R, et al. DHA attenuates postprandial hyperlipidemia via activating PPARa in intestinal epithelial cells. J Lipid Res. 2013;54(12):3258-68.

17. Lambert JE, Parks EJ. Postprandial metabolism of meal triglyceride in humans. Biochim Biophys Acta. 2012;1821(5):721-6.

18. Xu T, Li J, Zou J, Qiu B, Liu W, Lin X, et al. Rat Small Intestinal Mucosal Epithelial Cells Absorb Dietary 1,3-Diacylglycerol Via Phosphatidic Acid Pathways. Lipids. 2018;53(3):335-44.

19. Iena FM, Lebeck J. Implications of Aquaglyceroporin 7 in Energy Metabolism. Int J Mol Sci. 2018;19(1):154.

20. Laforenza U, Gastaldi G, Grazioli M, Cova E, Tritto S, Faelli A, et al. Expression and immunolocalization of aquaporin-7 in rat gastrointestinal tract. Biol Cell. 2005;97(8):605-13.

21. Dávalos-Salas $\mathrm{M}$, Montgomery MK, Reehorst $\mathrm{CM}$, Nightingale $\mathrm{R}, \mathrm{Ng} \mathrm{I}$, Anderton $\mathrm{H}$, et al. Deletion of intestinal Hdac3 remodels the lipidome of enterocytes and protects mice from diet-induced obesity. 
Nat Commun. 2019;10(1):5291.

22. Nakamura MT, Yudell BE, Loor JJ. Regulation of energy metabolism by long-chain fatty acids. Prog Lipid Res. 2014;53:124-44.

23. Watkins PA, Ellis JM. Peroxisomal acyl-CoA synthetases. Biochim Biophys Acta. 2012;1822(9):1411-20.

24. Kuwata H, Nakatani E, Shimbara-Matsubayashi S, Ishikawa F, Shibanuma M, Sasaki Y, et al. Longchain acyl-CoA synthetase 4 participates in the formation of highly unsaturated fatty acid-containing phospholipids in murine macrophages. Biochim Biophys Acta Mol Cell Biol Lipids. 2019;1864(11):1606-18.

25. Dai J, Liang K, Zhao S, Jia W, Liu Y, Wu H, et al. Chemoproteomics reveals baicalin activates hepatic CPT1 to ameliorate diet-induced obesity and hepatic steatosis. Proc Natl Acad Sci U S A. 2018;115(26):E5896-905.

26. Schönfeld P, Wojtczak L. Short- and medium-chain fatty acids in energy metabolism: the cellular perspective. J Lipid Res. 2016;57(6):943-54.

27. He Y, Yang T, Du Y, Qin L, Ma F, Wu Z, et al. High fat diet significantly changed the global gene expression profile involved in hepatic drug metabolism and pharmacokinetic system in mice. Nutr Metab (Lond). 2020;17:37.

28. Post SM, de Crom R, van Haperen R, van Tol A, Princen HM. Increased fecal bile acid excretion in transgenic mice with elevated expression of human phospholipid transfer protein. Arterioscler Thromb Vasc Biol. 2003;23(5):892-7.

29. Sztalryd C, Brasaemle DL. The perilipin family of lipid droplet proteins: Gatekeepers of intracellular lipolysis. Biochim Biophys Acta Mol Cell Biol Lipids. 2017;1862(10 Pt B):1221-32.

30. Sohn JH, Lee YK, Han JS, Jeon YG, Kim JI, Choe SS, et al. Perilipin 1 (Plin1) deficiency promotes inflammatory responses in lean adipose tissue through lipid dysregulation. J Biol Chem. 2018;293(36):13974-88.

31. Smetnev S, Klimushina M, Kutsenko V, Kiseleva A, Gumanova N, Kots A, et al. Associations of SNPs of the ADIPOQ Gene with Serum Adiponectin Levels, Unstable Angina, and Coronary Artery Disease. Biomolecules. 2019;9(10):537.

32. Obeid S, Wankell M, Charrez B, Sternberg J, Kreuter R, Esmaili S, et al. Adiponectin confers protection from acute colitis and restricts a B cell immune response. J Biol Chem. 2017;292(16):6569-82.

33. Phua T, Sng MK, Tan EH, Chee DS, Li Y, Wee JW, et al. Angiopoietin-like 4 Mediates Colonic Inflammation by Regulating Chemokine Transcript Stability via Tristetraprolin. Sci Rep. 2017;7:44351.

34. Liao C, Li M, Li X, Li N, Zhao X, Wang X, et al. Trichothecin inhibits invasion and metastasis of colon carcinoma associating with SCD-1-mediated metabolite alteration. Biochim Biophys Acta Mol Cell Biol Lipids. 2020;1865(2):158540.

35. Ducheix S, Peres C, Härdfeldt J, Frau C, Mocciaro G, Piccinin E, et al. Deletion of Stearoyl-CoA Desaturase-1 From the Intestinal Epithelium Promotes Inflammation and Tumorigenesis, Reversed by 
Dietary Oleate. Gastroenterology. 2018;155(5):1524-38.e9.

36. Renner SW, Walker LM, Forsberg LJ, Sexton JZ, Brenman JE. Carbonic anhydrase III (Car3) is not required for fatty acid synthesis and does not protect against high-fat diet induced obesity in mice. PLoS One. 2017;12(4):e0176502.

37. Supuran CT, Vullo D, Manole G, Casini A, Scozzafava A. Designing of novel carbonic anhydrase inhibitors and activators. Curr Med Chem Cardiovasc Hematol Agents. 2004;2(1):49-68.

38. Aspatwar A, Tolvanen ME, Parkkila S. An update on carbonic anhydrase-related proteins VIII, X and XI. J Enzyme Inhib Med Chem. 2013;28(6):1129-42.

39. Hilvo M, Innocenti A, Monti SM, De Simone G, Supuran CT, Parkkila S. Recent advances in research on the most novel carbonic anhydrases, CA XIII and XV. Curr Pharm Des. 2008;14(7):672-8.

40. Endeward V, Kleinke T, Gros G. Carbonic anhydrase in the gastrointestinal mucus of mammalspossible protective role against carbon dioxide. Comp Biochem Physiol A Mol Integr Physiol. 2003;136(2):281-7.

41. Tillman JB, Dhahbi JM, Mote PL, Walford RL, Spindler SR. Dietary calorie restriction in mice induces carbamyl phosphate synthetase I gene transcription tissue specifically. J Biol Chem. 1996;271(7):3500-6.

42. Saarnio J, Parkkila S, Parkkila AK, Waheed A, Karttunen T, Sly WS. Cell-specific expression of mitochondrial carbonic anhydrase in the human and rat gastrointestinal tract. J Histochem Cytochem. 1999;47(4):517-24.

43. Scott KP, Gratz SW, Sheridan PO, Flint HJ, Duncan SH. The influence of diet on the gut microbiota. Pharmacol Res. 2013;69(1):52-60.

44. Weinberg SE, Sena LA, Chandel NS. Mitochondria in the regulation of innate and adaptive immunity. Immunity. 2015;42(3):406-17.

45. Turner N, Heilbronn LK. Is mitochondrial dysfunction a cause of insulin resistance? Trends Endocrinol Metab. 2008;19(9):324-30.

46. Meng L, Yang X, Xie X, Wang M. Mitochondrial. NDUFA4L2 protein promotes the vitality of lung cancer cells by repressing oxidative stress. Thorac Cancer. 2019;10(4):676-85.

47. Nie K, Li J, He X, Wang Y, Zhao Q, Du M, et al. COX6B2 drives metabolic reprogramming toward oxidative phosphorylation to promote metastasis in pancreatic ductal cancer cells. Oncogenesis. 2020;9(5):51.

48. Li WX, Li GH, Tong X, Yang PP, Huang JF, Xu L, et al. Systematic metabolic analysis of potential target, therapeutic drug, diagnostic method and animal model applicability in three neurodegenerative diseases. Aging. 2020;12(10):9882-914.

49. Kane PM. Targeting reversible disassembly as a mechanism of controlling V-ATPase activity. Curr Protein Pept Sci. 2012;13(2):117-23.

50. Fuhrmann DC, Brüne B. Mitochondrial composition and function under the control of hypoxia. Redox Biol. 2017;12:208-15. 
51. Pajuelo Reguera D, Čunátová K, Vrbacký M, Pecinová A, Houštěk J, Mráček T, et al. Cytochrome $C$ Oxidase Subunit 4 Isoform Exchange Results in Modulation of Oxygen Affinity. Cells. 2020;9(2):443.

52. Evans AM, Mahmoud AD, Moral-Sanz J, Hartmann S. The emerging role of AMPK in the regulation of breathing and oxygen supply. Biochem J. 2016;473(17):2561-72.

53. Fischer B, Schöttl T, Schempp C, Fromme T, Hauner H, Klingenspor M, et al. Inverse relationship between body mass index and mitochondrial oxidative phosphorylation capacity in human subcutaneous adipocytes. Am J Physiol Endocrinol Metab. 2015;309(4):E380-7.

54. Clemmensen C, Müller TD, Woods SC, Berthoud HR, Seeley RJ, Tschöp MH. Gut-Brain Cross-Talk in Metabolic Control. Cell. 2017;168(5):758-74.

55. Guan X. The CNS glucagon-like peptide-2 receptor in the control of energy balance and glucose homeostasis. Am J Physiol Regul Integr Comp Physiol. 2014;307(6):R585-96.

\section{Figures}

a
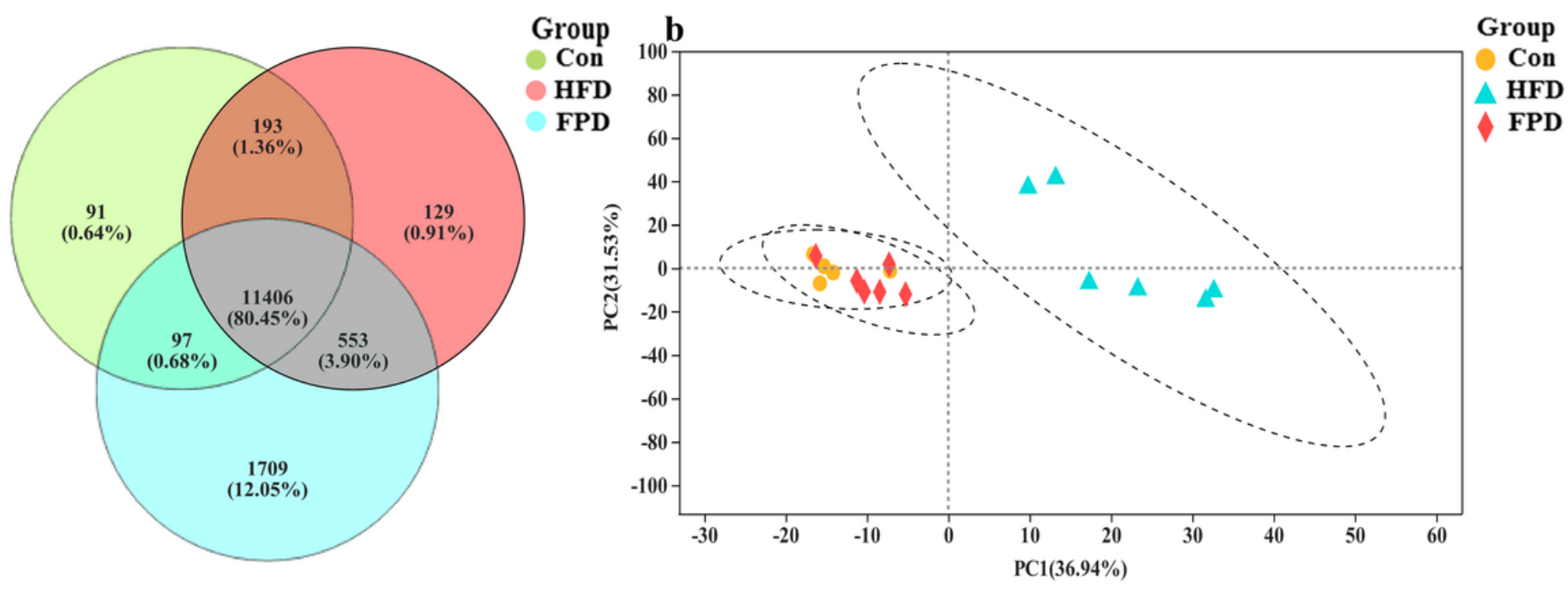

\section{Figure 1}

Analysis of the relationship between three groups. a, Venn diagram Venn; b, PCA analysis. Group Con indicates rats fed D12450B during the obesity model building and AIN-93M thereafter. Group HFD indicates rats fed D12492 during model building and AIN-93M thereafter. Group FPD indicates rats fed D12492 during model building and an FP containing diet thereafter. 

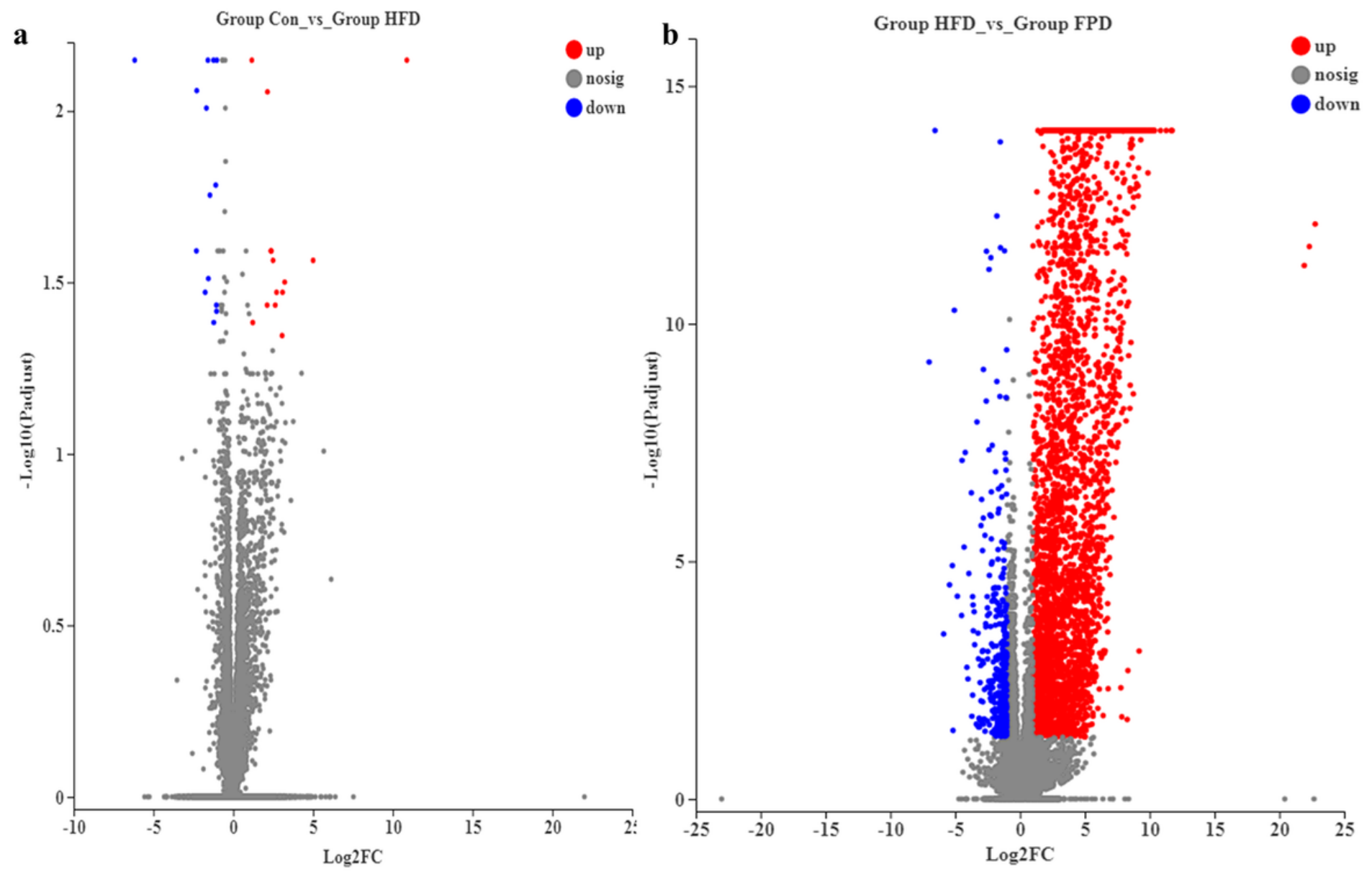

Figure 2

DEGs between different treatment groups. The horizontal axis indicates expression changes (log) of the genes in different treatment groups while the vertical axis shows the differences of gene expression. The discrepancy was more significant with smaller $p$-values and bigger - $\log 10$ (adjusted p-value). Splashes were for different genes, among which grey dots were genes with no significant discrepancy, red dots were genes significantly up-regulated and blue dots were significantly down-regulated genes. Abbreviations: DEGs, differentially expressed genes; Group Con indicates rats fed D12450B during the obesity model building and AIN-93M thereafter. Group HFD indicates rats fed D12492 during model building and AIN-93M thereafter. Group FPD indicates rats fed D12492 during model building and an FP containing diet thereafter. 


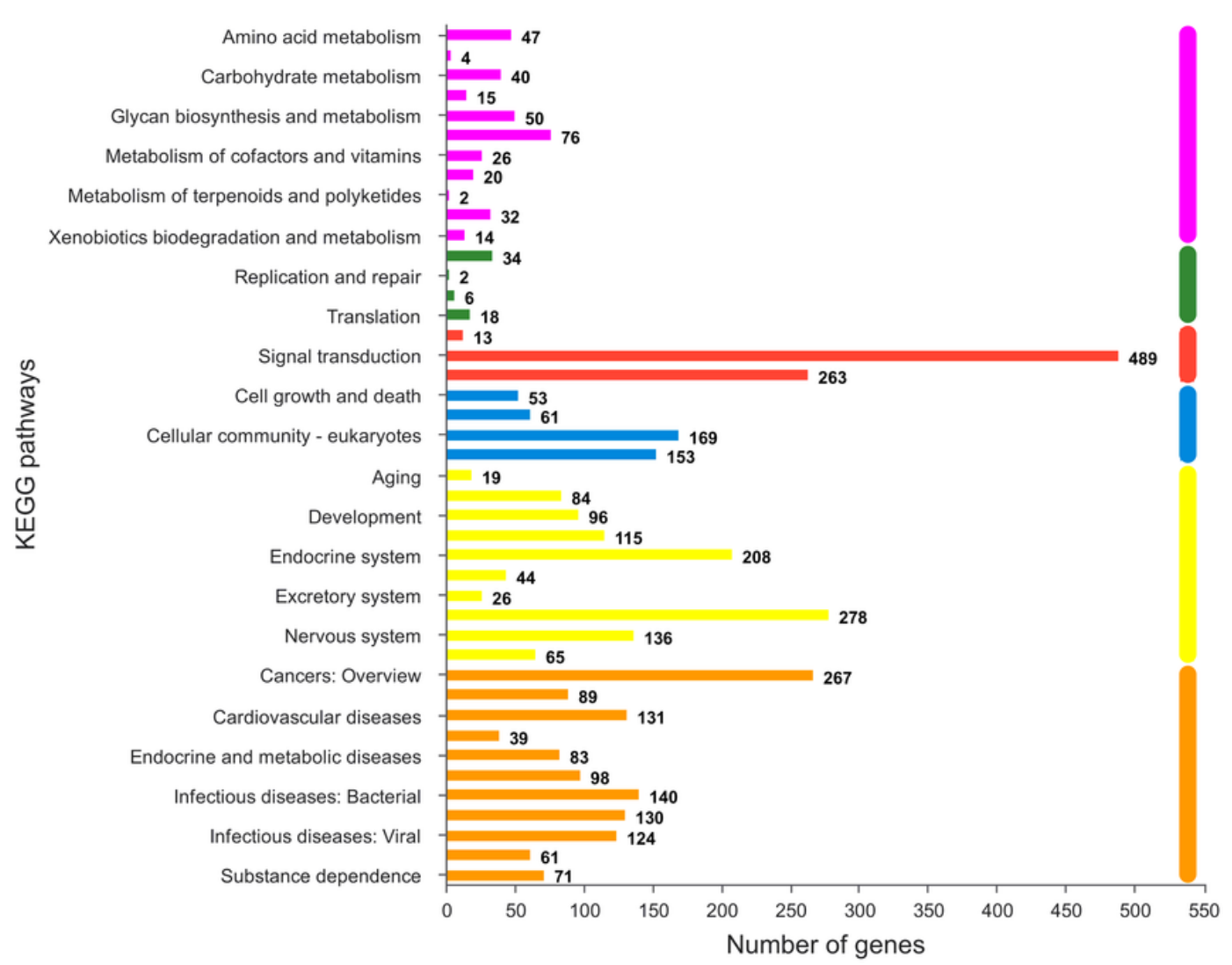

Metabolism

Genetic Information Processing

Environmental Information Processing

Cellular Processes

Organismal Systems

Human Diseases

\section{Figure 3}

KEGG function classification of DEGs in Group HFD and Group FPD Abbreviations: DEGs, differentially expressed genes; KEGG, Kyoto Encyclopedia of Genes and Genomes. Group HFD indicates rats fed D12492 during model building and AIN-93M thereafter. Group FPD indicates rats fed D12492 during model building and an FP containing diet thereafter. 


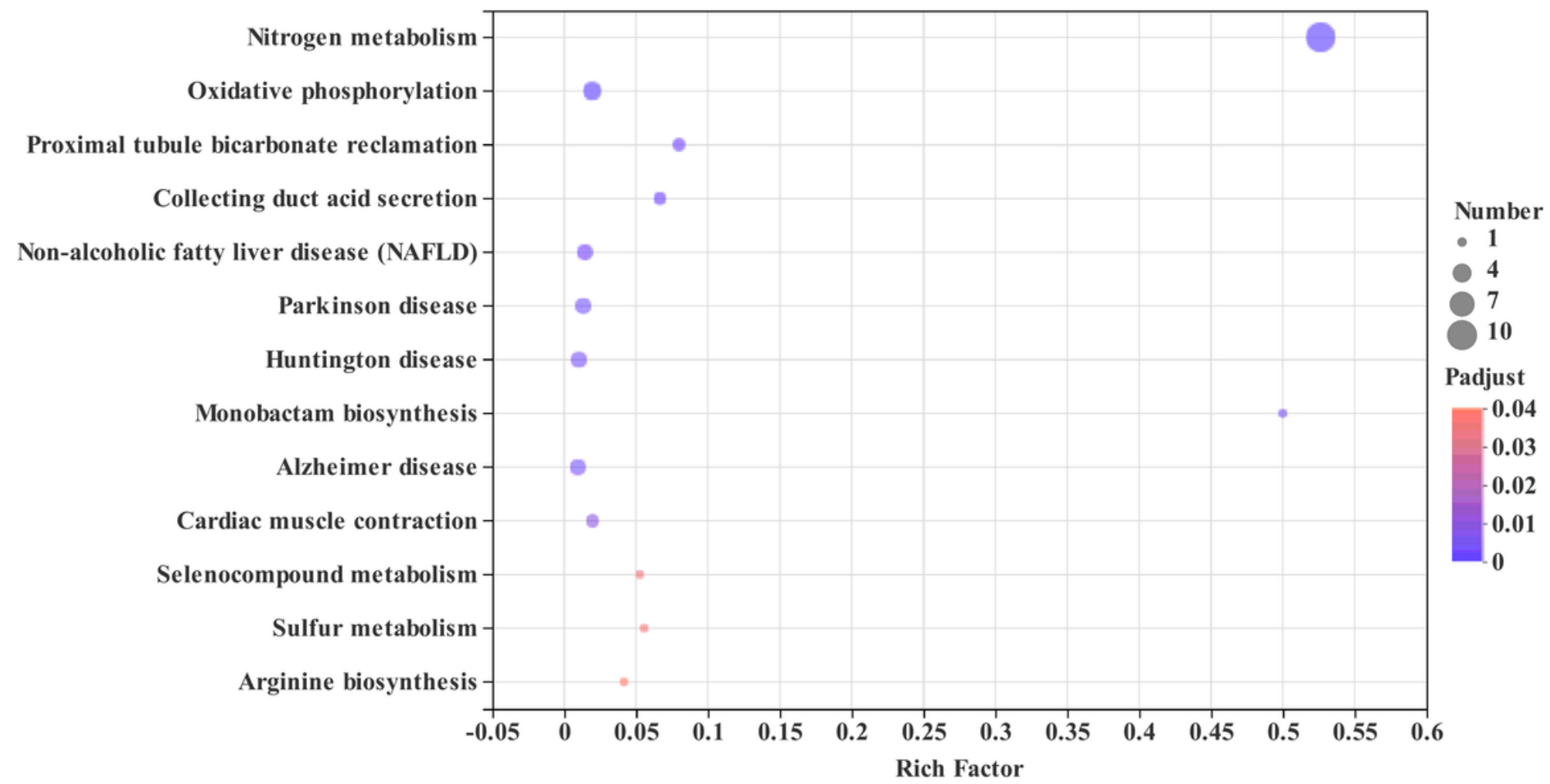

\section{Figure 4}

Bubble diagram of 15 DEGs between Group HFD and Group FPD. The vertical axis indicates KEGG pathway and the horizontal axis represents the Rich factor. The enrichment degree was stronger with a bigger Rich factor. The size of dots indicates the number of genes. Abbreviations: DEGs, differentially expressed genes; KEGG, Kyoto Encyclopedia of Genes and Genomes. Group HFD indicates rats fed D12492 during model building and AIN-93M thereafter. Group FPD indicates rats fed D12492 during model building and an FP containing diet thereafter. 


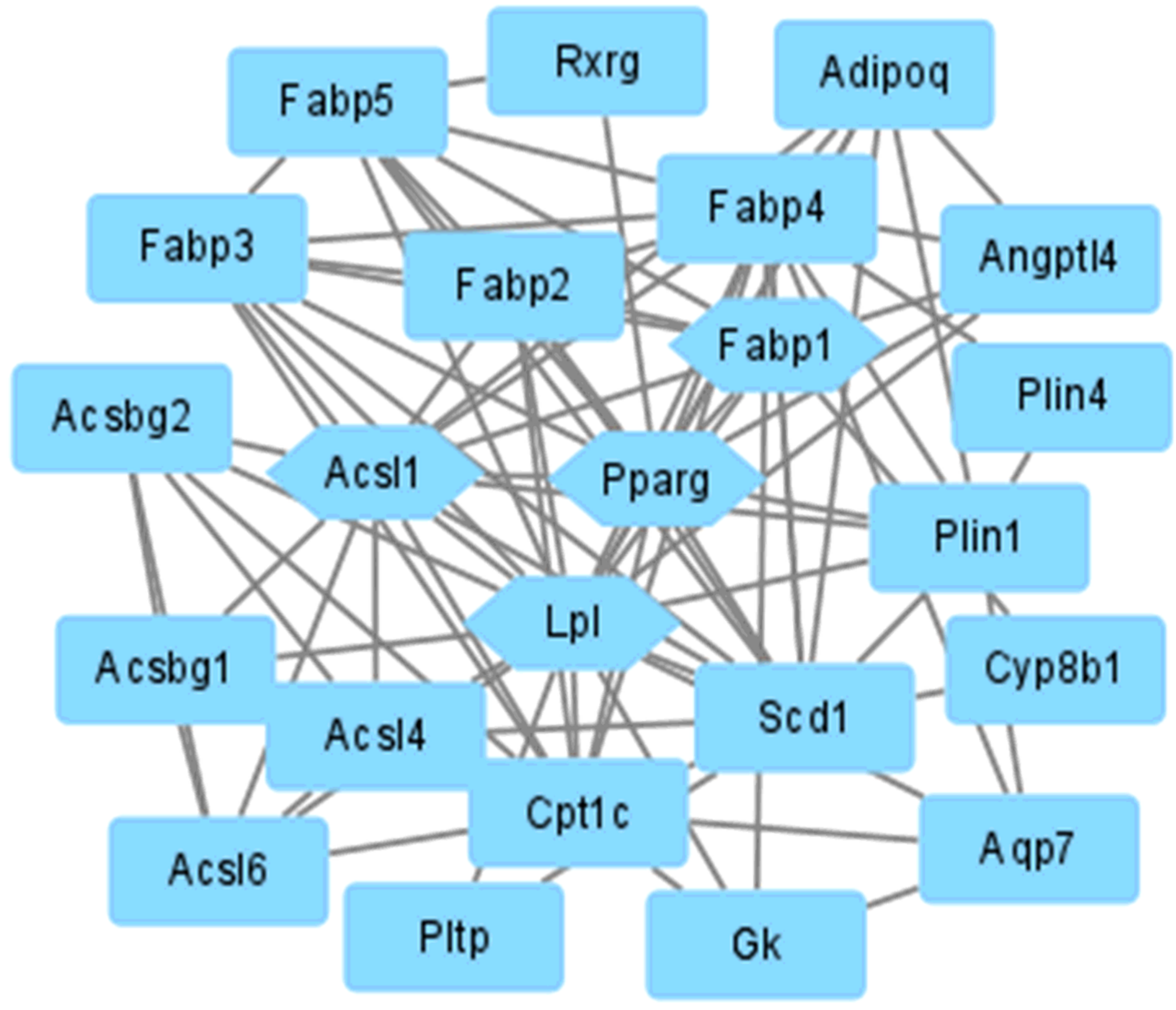

Figure 5

The PPI network of 23 DEGs in PPAR signaling pathway. Nodes stand for the proteins (genes), and edges stand for the interactions of proteins. Abbreviations: DEGs, differentially expressed genes; PPI, ProteinProtein Interaction. 

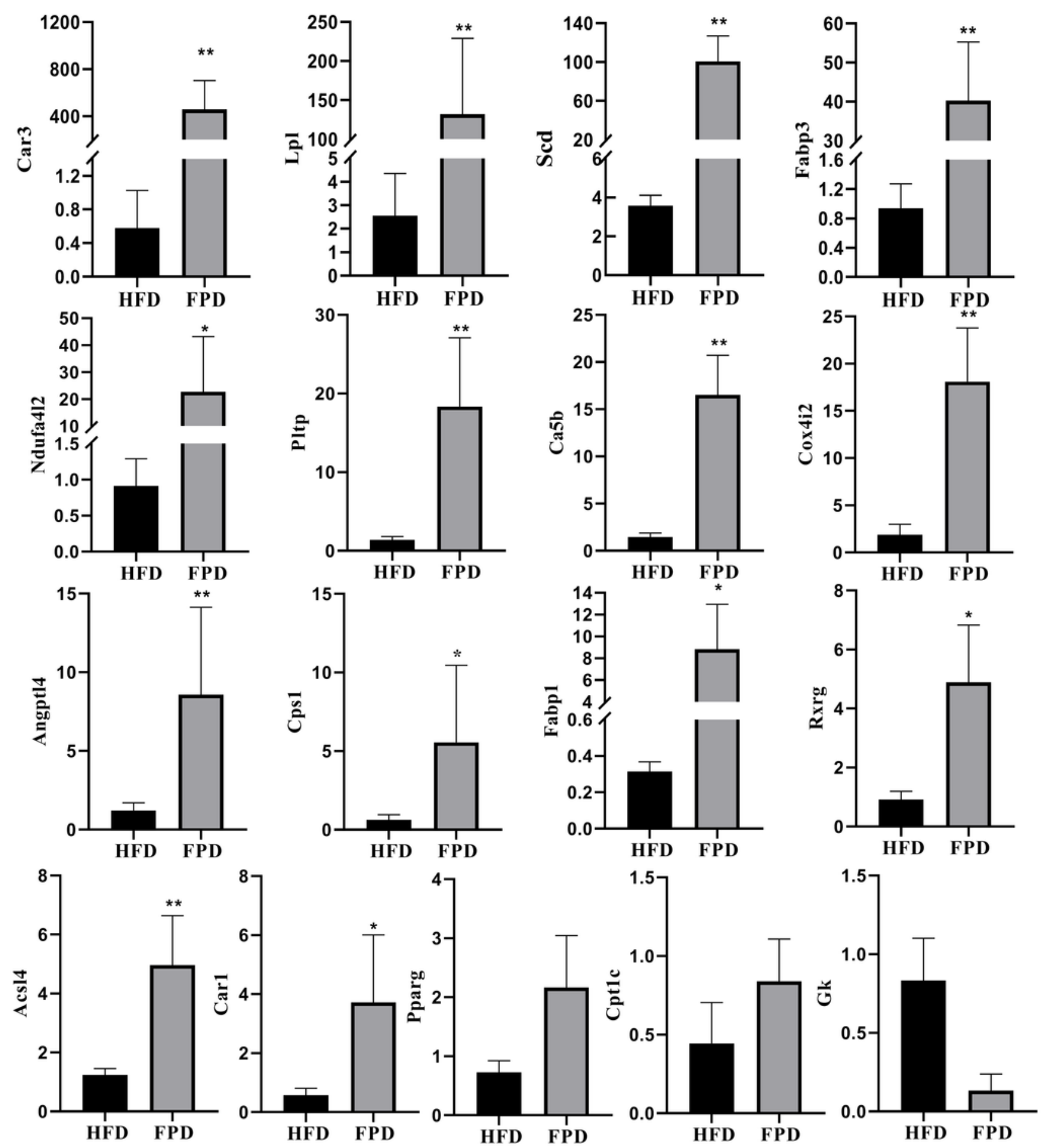

Figure 6

The qRT-PCR verification for RNA-Seq analysis. * * means $\mathrm{P}<0.01 .{ }^{*}$ means $\mathrm{P}<0.05$. 
Fatty acid

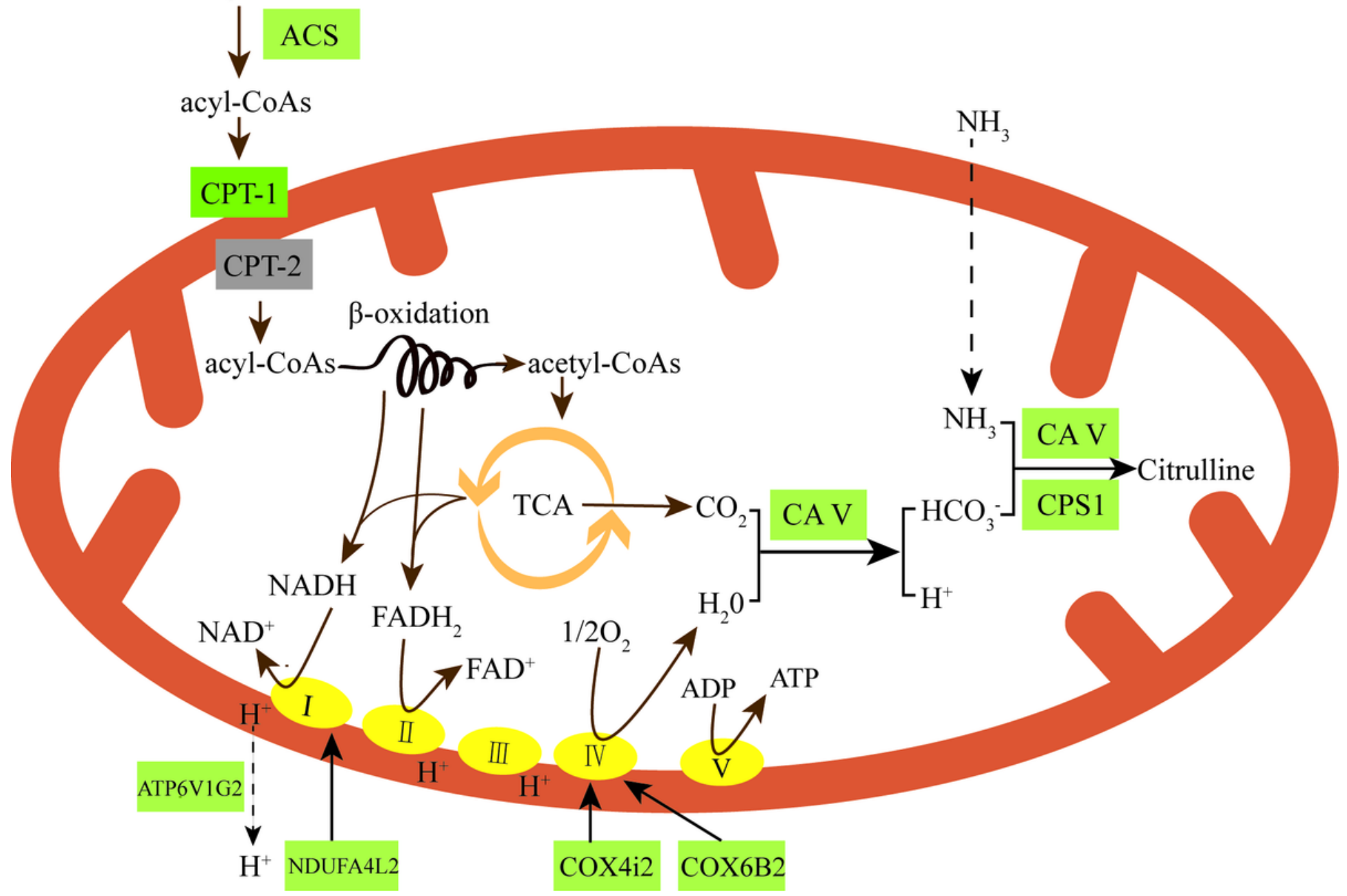

Figure 7

Illustration of potential regulatory mechanism involved in mitochondrial energy metabolism of FP intervention in the colonic epithelial cell. FP intervention upregulated ACS and CPT-1, promoting the transformation of FA to acyl-CoAs and the transfer into mitochondria for $\beta$ - oxidation and TCA cycle. Then, $\mathrm{CO} 2, \mathrm{NADH}$ and FADH2 were generated. FA intervention upregulated the relative proteins of complexes I and IV, promoting oxidative phosphorylation and generated $\mathrm{H} 2 \mathrm{O}$. CA V catalyzed $\mathrm{H} 2 \mathrm{O}$ and $\mathrm{CO} 2$ into $\mathrm{HCO} 3-$, and then co-catalyzed with $\mathrm{CPS} 1$ for the transformation of $\mathrm{NH} 3$ and $\mathrm{HCO} 3-$ to Citrulline. Therefore, the anti-obesity effect of FP intervention functioned through promoting the catabolism of FA to facilitate lipid metabolism and energy metabolism. Note that rectangles in green indicate upregulation of proteins in group FPD compared with group HFD, while that in grey indicates no significantly alteration. Group HFD indicates rats fed D12492 during model building and AIN-93M thereafter. Group FPD indicates rats fed D12492 during model building and an FP containing diet thereafter.

\section{Supplementary Files}

This is a list of supplementary files associated with this preprint. Click to download. 
- SupplementaryTableS1.docx

Page 25/25 